\title{
A experiência de um estágio internacional de Cuidados de Saúde Primários em Boston, EUA
}

Ana Luísa Neves*

\section{RESUMO}

Este artigo pretende transmitir a experiência de um estágio internacional que decorreu em Junho de 2010, no Union Square Family Health (USFH) (Somerville, Boston), uma unidade de cuidados de saúde primários afiliada da Harvard Medical School e da Tufts University of Family Medicine.

Este estágio constituiu uma oportunidade de contactar com a prática clínica diária da Medicina Geral e Familiar nos EUA, nomeadamente ao nível da participação em consultas programadas e não-programadas, sessões de educação para a saúde e de programas de redução do risco cardiovascular, observação e aquisição de competências em técnicas no âmbito da pequena cirurgia, ginecologia em Cuidados de Saúde Primários e infiltrações articulares. Foi também possível contactar com o Programa de Internato de Medicina Geral e Familiar da Tufts University, ao nível da participação em videoconferências e seminários, assim como na observação tutorizada de pacientes e respectiva discussão dos casos.

Através da descrição das actividades desenvolvidas e das especificidades com que contactou durante o estágio supracitado, a autora pretende estabelecer uma reflexão crítica sobre a sua experiência.

Como conclusão, são discutidas as vantagens inerentes à realização de um estágio internacional, entre as quais salienta o contacto com novas ideias e diferentes formas de trabalho, a exposição a novas práticas (e o seu uso como ferramenta de melhoria contínua), a aquisição de novas capacidades de comunicação, o estabelecimento de contactos profissionais e a valorização das qualidades do nosso próprio sistema.

Palavras-chave (DeCS): Intercâmbio Educacional Internacional; Cuidados de Saúde Primários.

\section{INTRODUÇÃO}

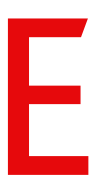

ste artigo pretende transmitir a experiência de um estágio internacional no Union Square Family Health (USFH) (Somerville, Boston), uma unidade de cuidados de saúde primários afiliada da Harvard Medical Schoole da Tufts University of Family Medicine.

A internacionalização é uma tendência crescente em Cuidados de Saúde Primários, seja através da participação em congressos ou conferências, da submissão de artigos a revistas internacionais, ou da realização de estágios como parte do plano de formação ou extra-curriculares. As vantagens de um contacto com a realidade dos Cuidados de Saúde Primários num contexto di-

*Internato Médico de Medicina Geral e Familiar, Unidade de Saúde Familiar Camélias - ACES Gaia VIII. Programa Doutoral em Medicina, Faculdade de Medicina da Universidade do Porto. ferente representa uma oportunidade privilegiada para a aquisição de novos conhecimentos e competências, para o contacto com novas formas de trabalho e, inclusivamente, para a abertura de hipóteses de colaboração num universo cada vez mais global. Por todos estes motivos, a autora considerou que a realização de um estágio internacional poderia constituir uma mais-valia na sua formação médica específica.

O estágio decorreu durante o mês de Junho de 2010, sob a orientação da Prof. RachelWheeler, Médica de Família no USFH. Constituiu assim uma oportunidade de contacto com a prática clínica diária da Medicina Geral e Familiar nos EUA, na qual foi possível assistir e participar nas consultas médicas, sessões de educação para a saúde e reuniões médicas, assim como colaborar nas actividades de outros profissionais de saúde (assistente social, nutricionista, health advisor). Por outro lado, 
sendo um centro afiliado a duas faculdades de Medicina, o estágio no USFH permitiu ainda um contacto próximo com o programa de internato local de Medicina Geral e Familiar, como seguidamente se descreve.

\section{O CENTRO DE ACOLHIMENTO - UNION SQUARE FAMILY HEALTH (USFH)}

O USFH integra-se na Cambridge Health Alliance (CHA), um sistema de saúde regional que presta cuidados de saúde à população de Cambridge, Somerville e da grande área metropolitana de Boston. O CHA inclui três hospitais de referência (Cambridge Hospital, Somerville Hospital e Whidden Memorial Hospital), o departamento de Saúde Pública de Cambridge, uma rede de mais de 20 unidades de cuidados de saúde primários e clínicas de outras especialidades médicas.

A comunidade abrangida por este centro de saúde é bastante diversa sob o ponto de vista étnico e cultural - resultado do grande impacto da imigração nesta área, principalmente de indivíduos provenientes da América central e do sul (Brasil, Porto Rico, El Salvador, Haiti), mas também portugueses (principalmente dos Açores) e de outras nacionalidades (Índia, Paquistão, China, Tailândia). Por isso constitui um desafio constante para os profissionais de saúde lidar com uma população ambivalente entre o desejo de se integrar na comunidade e de não perder a sua própria identidade mas também o desafio sob o ponto de vista médico de empatizar, comunicar e educar uma população com tão ampla diversidade cultural.

A equipa do USFH é constituída por uma equipa médica, de enfermagem e de medical assistants. Os medical assistants são o primeiro contacto do doente com os serviços de saúde e têm funções bastante polivalentes (desde o acolhimento dos doentes, a avaliação dos parâmetros biométricos e sinais vitais e a colheita de sangue ou outros produtos biológicos), que desenvolvem de forma rotativa, segundo um esquema de turnos. Assim, o pessoal de enfermagem fica disponível para outro tipo de actividades, tais como a triagem das consultas não-programadas, a renovação de prescrições crónicas (após autorização médica) e a resolução de problemas que não necessitem da intervenção directa do médico.

A equipa conta ainda com outros técnicos de saúde, nomeadamente uma psicóloga, uma nutricionista, uma assistente social e vários health advisors. Os health advisors são técnicos de saúde que se dedicam exclusivamente a actividades preventivas e que consultam de forma sistemática a população abrangida para detectar problemas de saúde ou hábitos de risco passíveis de serem melhorados através de um programa activo de prevenção (por exemplo, obesidade, tabagismo, consumo de álcool ou drogas, diabetes, hipertensão, dislipidémia). $\mathrm{O}$ health advisor participa também na dinamização de actividades e sessões de educação para a saúde e verifica de forma sistemática o programa de rastreios na lista de utentes, convocando-os sempre que necessário para actualização.

A CHA (da qual o USFH faz parte) tem uma organização própria, funcionando como uma unidade local de saúde pública (vide supra). Cada médico tem uma lista de utentes que, no caso deste centro, era constituída por cerca de 1500 utentes. As consultas médicas podem ser programadas ou não programadas (walkins). As consultas não-programadas são triadas por um enfermeiro, que determina a urgência do caso e orienta para uma consulta médica urgente no próprio dia ou, se tal não for necessário, para uma consulta programada a posteriori. Em alguns casos, se for apenas necessário aconselhamento ou outros procedimentos não-médicos, o doente é assistido na consulta de enfermagem.

Em relação aos exames auxiliares de diagnóstico, que podem ser pedidos pelos Médicos de Família ou pelas Nurse Assistants, existem também algumas diferenças. As colheitas são feitas directamente no centro de saúde e as amostras enviadas ao final do dia para o hospital de referência (neste caso o Somerville Hospital), para serem analisadas. Os resultados são depois enviados através do sistema informático comum para o processo do doente. Os restantes exames são marcados através do mesmo sistema de informação e o doente é informado da hora a que deve comparecer no hospital de referência.

Para o registo dos dados do utente é utilizado um sistema informático (EPIC ${ }^{\circledR}$ ) comum aos cuidados de saúde primários e ao hospital. O sistema funciona na base da introdução dos dados numa lista de verificação dos mesmos, a partir da qual é gerado um documento-tipo em texto, de acordo com as queixas referida pelo doente. Este sistema permite uma articulação directa 
com o hospital de referência, ao nível da marcação de consultas, mas também da consulta de relatórios, resultados de exames auxiliares de diagnóstico e informação hospitalar de retorno. Por fim, este sistema integra uma extensa base de dados com outros documentos, tais como conselhos práticos sobre patologias prevalentes em cuidados de saúde primários, que podem ser impressos e entregues ao doente no final da consulta, como complemento informativo.

O doente pode aceder ao seu processo clínico após dar o seu consentimento, através de um sistema designado MyChart $\AA^{\circledR}$, sendo-lhe atribuído um login e password. Acedendo a um site próprio pode consultar o seu processo clínico, mas também enviar mensagens ao seu Médico de Família para esclarecimento de dúvidas ou pedidos de renovação de medicação. A tendência do sistema é eminentemente electrónica: a prescrição é feita através do sistema informático e a ordem é enviada directamente à farmácia (previamente designada pelo paciente como a sua farmácia habitual), na qual o doente levantará a medicação sem qualquer tipo de papel.

\section{DESCRIÇÃO DAS ACTIVIDADES CLÍNICAS DESENVOLVIDAS E ALGUMAS PARTICULARIDADES DOS CUIDADOS PRIMÁRIOS NA CHA}

Durante este estágio a autora participou nas consultas médicas de doentes com problemas médicos e das diversas faixas etárias (sendo de notar que o atendimento programado dos médicos do USFH não está organizado por grupos vulneráveis ou de risco). Após as consultas, os casos e respectiva orientação eram discutidos, e os dados registados.

Ainda no USFH, a autora participou em sessões de educação para a saúde desenvolvidas por equipas multidisciplinares, no âmbito de patologias crónicas (tais como sessões sobre nutrição da pessoa com diabetes, de acompanhamento do programa de cessação tabágica, de aconselhamento sobre $o$ aleitamento materno, e de informação aos pais sobre vacinação infantil). $O$ CHA desenvolve actualmente um programa de redução de risco cardiovascular, para o qual são convocados os doentes considerados de maior risco para sessões periódicas. É feita a identificação dos factores modificáveis nestes e é proposto um plano de redução do risco em função de cada caso, que é depois monitorizado.
Neste contexto, participou na realização destas sessões, acompanhada pelo health advisor da unidade.

Por último, a participação em reuniões médicas e nas consultas de outros profissionais de saúde (assistente social, nutricionista, health advisor), permitiu um contacto com a organização inter-disciplinar da equipa e com as actividades de outros profissionais de saúde.

Apesar das diferenças estruturais entre os sistemas de saúde nos EUA e em Portugal, a prática diária da Medicina Geral e Familiar encontra também múltiplos pontos de contacto ao nível dos conceitos básicos - colheita da história clínica, realização do exame objectivo, orientação diagnóstica, escolha terapêutica e normas de orientação.

Existem, no entanto, algumas particularidades que merecem consideração. Contrariamente ao nosso sistema de vigilância partilhada da gravidez, o Médico de Família pode, se assim entender, fazer o acompanhamento da gravidez desde a pré-concepção até à sua fase final. Durante o programa de internato existe formação prática específica no âmbito da assistência da mulher no parto, e grande parte dos Médicos de Família realizam eles próprios os partos das suas utentes no hospital. No âmbito da saúde da mulher, foi proposto à autora assistir às consultas da Dra. Sabrina Selim (Broadway Health Center, CHA), que se dedica com especial interesse a esta área. Para além da participação nas consultas, foi possível observar a realização de algumas técnicas ginecológicas (colposcopias, biópsias de endométrio, observação de exsudados ao microscópio para diagnóstico imediato).

Por outro lado, existe também a generalização da realização de alguns procedimentos em cuidados de saúde primários - desde técnicas de pequena cirurgia como excisão de nevos, realização de biópsias cutâneas e crioterapia, até à realização de infiltrações articulares. Neste âmbito, foi sugerida a realização de alguns períodos do estágio no Ball Square Family Health, sob supervisão do Dr. Michael Coffey, durante os quais foi possível observar as técnicas supracitadas.

Por último, uma nota relativamente ao facto de, no momento de realização do estágio, as seguradoras desempenharem um papel muito importante no sistema de saúde americano, o que introduz algumas particularidades na prática diária. Em primeiro lugar, esta ca- 
racterística do sistema tem um impacto grande na adesão terapêutica, uma vez que é frequente os doentes decidirem que não cumprirão determinada terapêutica ou procedimento diagnóstico porque a seguradora não comparticipa, ou porque esta comparticipação é insuficiente. Em segundo lugar, mesmo numa consulta dita de «rotina», cada um dos exames passíveis de serem pedidos é extensamente discutido com o doente (mesmo cada um dos componentes do estudo analítico, tais como função renal, função hepática, hemograma), sendo a partir desta discussão que as prioridades se vão definindo e o plano negociado.

\section{O PROGRAMA DE INTERNATO DE MEDICINA GERAL E FAMILIAR}

O primeiro ano de internato é fundamentalmente hospitalar, no qual os internos desenvolvem actividades sob supervisão de especialistas. Durante este primeiro ano pretende-se que tomem contacto com a realidade dos cuidados de saúde secundários, mas também que adquiram competências iniciais no âmbito da Medicina Geral e Familiar, mantendo para o efeito um número variável de períodos de ligação a uma Unidade de Cuidados de Saúde Primários.

Como um interno de segundo ano, a carga horária na Unidade de Cuidados de Saúde Primários vai aumentando progressivamente. Para além disso, os internos participam em algumas rotações hospitalares que se focam fundamentalmente na aquisição de competências técnicas (Quadro I).

Finalmente, durante o terceiro ano, as competências necessárias à prática da Medicina Geral e Familiar são refinadas, de forma a preparar a transição para a prática autónoma. No centro que a autora teve oportunidade de visitar, o Malden Family Health Center, existem vários gabinetes para os internos realizarem as consultas e uma biblioteca onde os orientadores se encontram, a partir da qual vão verificando os registos informáticos realizados pelos internos ao longo da consulta. No final da consulta, os internos deslocamse a esta sala para discutir com os orientadores cada caso e, se necessário, confirmarem juntos algum achado do exame físico. Os orientadores têm turnos rotativos no programa de residência, e assim cada interno contacta com diferentes formas de trabalho no entanto, isto constitui, como os próprios conside- ram, uma limitação ao nível da continuidade dos cuidados prestados ao doente.

Durante o programa de formação, os internos são ainda encorajados a fazer estágios em outros centros internacionais (electives), em áreas pelas quais tenham especial interesse (Medicina Geral e Familiar, diabetes, geriatria, saúde da mulher, medicina rural).

Relativamente à formação teórica, na Tufts University Family Medicine Residency existem diariamente conferências organizadas pelo Programa de Internato e que são dinamizadas por Médicos de Família ou de outras especialidades. Nestas são discutidos temas de gestão da prática clínica, pediatria, saúde da mulher, geriatria, radiologia, dermatologia, entre outros. As sessões têm lugar numa das unidades de cuidados de saúde primários ou nos hospitais afiliados e são difundidas em vídeo-conferência para as restantes unidades. Decorrem normalmente ao início da manhã, segundo um plano previamente aprovado, do qual os internos tomam conhecimento (sendo a participação nas conferências oportunística, conforme as áreas nas quais consideram sentir mais lacunas).

Semanalmente, existe uma reunião sobre comunicação e medicina comportamental, um grupo Balint semanal dirigido aos internos do segundo e terceiro ano, focando-se essencialmente na discussão das experiências individuais problemáticas ou pacientes difíceis, e nas particularidades da relação médico-doente. Mensalmente, existe um Clube de Leitura e seminários de medicina baseada na evidência, nos quais todos os residentes e supervisores discutem e avaliam artigos científicos recentes.

Durante este estágio, foi permitido à autora um breve contacto com este programa de internato, durante dois períodos por semana. Foi-lhe assim possível participar nas videoconferências e nos seminários de medicina baseada na evidência, nos quais foram abordados temas diversos no âmbito da Medicina Geral e Familiar (discussão de casos clínicos; apresentações sobre temas diversos, tais como a detecção precoce de situações de violência familiar; conceitos sobre fiabilidade e confidencialidade dos registos clínicos electrónicos em Cuidados de Saúde Primários, discussão do modelo de cuidados Patient-Centered Medical Home). Por outro lado, durante um período por semana deslocou-se ao Malden Family Health Center, onde foi pos- 
sível participar de forma activa na observação de pacientes com os internos do programa de internato, assim como na posterior discussão dos mesmos com a Dra. Rachel Wheeler, que desempenhava funções de tutora neste centro.
Outras actividades desenvolvidas durante o internato

Como parte do programa, os internos devem desenvolver ainda um programa longitudinal de intervenção na comunidade em articulação com as instituições locais, durante o segundo e terceiro anos, sob supervisão

\begin{tabular}{|c|c|c|c|}
\hline & 1. ${ }^{\circ}$ Ano & 2. ${ }^{\circ}$ Ano & 3. ${ }^{\circ}$ Ano \\
\hline $\begin{array}{l}\text { Período de ligação à Unidade } \\
\text { de Cuidados de Saúde } \\
\text { Primários (por semana) }\end{array}$ & 1 a 5 sessões de meio dia & 2 a 7 sessões de meio dia & 2 a 8 sessões de meio dia \\
\hline Atendimento nocturno & $\begin{array}{l}1 \text { noite por mês, } 9 \text { meses por } \\
\text { ano }\end{array}$ & $\begin{array}{l}15 \text { fins de semana por ano } \\
6 \text { urgências de ginecologia/ } \\
\text { /obstetrícia }\end{array}$ & 15 fins de semana por ano \\
\hline Estágios (em semanas) & $\begin{array}{l}\text { Introdução à Medicina Geral } \\
\text { e Familiar - } 4 \\
\text { Medicina Interna, } \\
\text { internamento - } 12 \\
\text { Unidade de Cuidados } \\
\text { Intensivos - } 4 \\
\text { Obstetrícia - } 6 \\
\text { Pediatria, internamento - } 5 \\
\text { Pediatria comportamental/ } \\
\text { /desenvolvimento - } 3 \\
\text { Berçário - } 3 \\
\text { Psiquiatria - } 3 \\
\text { Cirurgia - } 5 \\
\text { Ginecologia- } 3\end{array}$ & $\begin{array}{l}\text { Medicina Interna, } \\
\text { internamento - } 6 \\
\text { Medicina Interna, sala de } \\
\text { urgência - } 4 \\
\text { Obstetrícia - } 6 \\
\text { Ortopedia e Medicina } \\
\text { Desportiva - } 6 \\
\text { Pediatria, consulta - } 4 \\
\text { Medicina Geral e Familiar - } 22\end{array}$ & $\begin{array}{l}\text { Medicina Interna, } \\
\text { internamento - } 6 \\
\text { Pediatria, sala de urgência - } 4 \\
\text { Estágio opcional - } 8 \\
\text { Medicina Geral e Familiar - } 30\end{array}$ \\
\hline Outros estágios longitudinais & Desenvolvimento profissional & $\begin{array}{l}\text { Procedimentos e Técnicas; } \\
\text { Geriatria; Subespecialidades } \\
\text { de Medicina Interna; Técnicas } \\
\text { de comunicação;Técnicas de } \\
\text { ensino }\end{array}$ & $\begin{array}{l}\text { Procedimentos e Técnicas; } \\
\text { Geriatria; Gestão da Prática } \\
\text { Clínica, Medicina Comunitária; } \\
\text { Liderança e eficácia organizativa; } \\
\text { Projecto académico }\end{array}$ \\
\hline $\begin{array}{l}\text { Cursos do } 1 .^{\circ} \text { Ano: Suporte } \\
\text { avançado de vida; Ressuscitação } \\
\text { neonatal; Advanced Cardiac } \\
\text { Life Support } \\
\text { Cursos do 2. } .^{\circ} \text { Ano: Advanced } \\
\text { Cardiac Life Support; Clinical } \\
\text { Training in Mind/Body } \\
\text { Medicine, (MGH and Harvard } \\
\text { Medical School) } \\
\text { Cursos do 3. Ano: Advanced } \\
\text { Cardiac Life Support }\end{array}$ & & & \\
\hline
\end{tabular}

*Adaptado de Tufts University Family Medicine Residency Curriculum ${ }^{1}$ 
dos orientadores. O tema do programa é escolhido pelos internos e deverá, preferencialmente, surgir como resposta às necessidades da comunidade numa área médica específica. Alguns exemplos incluem: o Sharewood Project (Free Medical Clinic for Homeless); Senior Citizen Community Lectures; Malden Public School Presentations; Breastfeeding Class e o Malden Public Library Annual Family Health Day.

A participação em actividades de investigação clínica não é obrigatória, mas é bastante estimulada. A ligação com o departamento de Family Medicine and Community Health da Tufts Universitye com a Harvard Medical School permite aos internos integrarem projectos destas instituições, nomeadamente no desenvolvimento de abordagens alternativas na prevenção da doença e prestação de cuidados de saúde, metodologias de análise de dados epidemiológicos e clínicos, nutrição e saúde pública. Por outro lado, podem ainda colaborar com a rede de investigação Family Practice Research Network (ReNet), que proporciona uma base de colaboração entre os vários departamentos de cuidados primários.

\section{REFLEXÕES FINAIS}

A importância da internacionalização é uma realidade crescente em todos os campos da ciência e a Medicina, em particular a Medicina Geral e Familiar, não escapa a essa tendência. ${ }^{2}$

As possibilidades são múltiplas, desde a participação em reuniões científicas (WONCA, ${ }^{3}$ European General Practice Research Network ${ }^{4}$ ), a colaboração em publicações internacionais, até à realização de estágios. Estes últimos podem consistir em iniciativas e acordos individuais ou estar inseridos em oportunidades estruturadas como o Programa Hippokrates, ${ }^{5}$ os programas de intercâmbio com o apoio da APMCG (por exemplo o GP Dutch trainee exchange program e o intercâmbio com o Royal College of Family Physicians). O Movimento Vasco da Gama ${ }^{6}$ pretende estabelecer uma rede de contactos, proporcionando uma melhoria do ensino, investigação e oportunidades de intercâmbio aos internos e jovens médicos de família.

A experiência de um estágio internacional no âmbito do internato de Medicina Geral e Familiar proporciona múltiplas vantagens, directas e indirectas, que concorrem para uma melhoria da prática clínica: o con- tacto com novas ideias e diferentes formas de trabalho, a exposição a novas práticas, o desenvolvimento do curriculum pessoal, a melhoria do uso de outros idiomas, a aquisição de outras capacidades de comunicação num contexto diferente e o estabelecimento de novos contactos profissionais. ${ }^{7}$ Para além disso, o contacto com uma realidade diferente permite detectar lacunas e novas possibilidades de melhoria contínua no serviço de origem, que beneficiará com novas ideias e práticas de uma realidade distinta, mas extrapolável. ${ }^{8}$ Permite ainda, sem dúvida, valorizar as nossas próprias qualidades, tantas vezes esquecidas no quotidiano. $\mathrm{O}$ contacto com uma realidade diferente proporciona não apenas uma nova visão médica, científica e profissional, mas também uma nova visão social, ${ }^{8}$ na qual se insere um maior conhecimento e uma tolerância mais ampla face à multiplicidade de contextos geográficos, étnicos e sócio-culturais do ser humano, essa descoberta última na qual assenta toda a nossa prática clínica.

\section{REFERÊNCIAS BIBLIOGRÁFICAS}

1. Tufts University Family Medicine Residency. Department of Family Medicine, Cambridhe Health Alliance, Cambridge, USA. Disponível em: http://www.tuftsfmr.org/fam_med_res.shtml [acedido em 09/12/ 2010].

2. Green $N$, Ansar M, Pettigrew L. A snapshot of general practice in Europe. Br J Gen Pract 2009 May; 59 (562): 375.

3. WONCA Europe 2010 Conference. SemFYC congresos, Barcelona. Disponível em: http://www.woncaeurope2010.org/ [acedido em 09/12/2010].

4. European General Practice Research Network (EGPRN). EGPRN, Maastricht.. Disponível em: http://egprn.org/web/ [acedido em 09/12/2010].

5. Hippokrates program. Vasco da Gama Movement (VdGM), Europe Council. Disponível em : http://www.vdgm.eu/index.cfm/spKey/exchange.hippohrates.what_is_hippokrates_html [acedido em 09/12/ 2010].

6. The Vasco da Gama Movement. The WONCA Europe Working Group for Young and Future General Practitioners, Europe Council. Disponível em: http://www.woncaeurope.org/Vasco\%20da\%20Gama\%20 Movement.htm [acedido em 09/12/2010].

7. Gavilán-Moral E, Jiménez de Gracia L, Olivero FJ, Arévalo de Miguel E, Claraco Vega LM. Rotaciones externas de residentes.AMF (Actualización en Medicina de Familia) 2006; 2 (8): 482-6.

8. Villanueva T. ¿Por qué es importante salir al extranjero durante la residencia en medicina de familia? ¿cómo lograrlo? Aten Primaria 2011 Mar; 43 (3): 159-60.

\section{AGRADECIMENTOS}

The author thanks Dr. Rachel Wheeler (Union Square Family Health, CHA), 
Dr. Michael Coffey (Family Medicine @Ball Square) and Dr. Sabrina Selim (Broadway Health Center, CHA) for their interest and support, and Prof. Dr. Richard Roberts, for his kind help connecting the author with the doctors cited above.

A autora agradece ainda o apoio da Coordenação do Internato de Medicina Geral e Familiar da Zona Norte, sem o qual a realização deste estágio não teria sido possível.
CONFLITOS DE INTERESSE

A autora declara não possuir quaisquer conflitos de interesses.

\author{
ENDEREÇO PARA CORRESPONDÊNCIA \\ Ana Luísa Neves \\ Unidade de Saúde Familiar Camélias \\ Rua João de Deus 98/106 \\ 4410-182 Vila Nova de Gaia \\ E-mail: ana.luisa.neves@gmail.com
}

Recebido em 07/09/2010

Aceite para publicação em 01/02/2011

\section{ABSTRACT}

\section{THE EXPERIENCE OF AN INTERNATIONAL MEDICAL INTERNSHIP IN PRIMARY CARE IN BOSTON, EUA}

This article aims to convey the experience of an internship program held in June 2010, at Union Square Family Health (USFH) (Somerville, Boston), a primary care unit affiliated to Harvard Medical School and Tufts University of Family Medicine.

Through the description of activities and circumstances of the aforementioned internship, the author intends to establish a critical reflection about her experience.

This internship was an opportunity to contact with the daily practice of a family doctor in the U.S., during which the author participated in consultations, health education and cardiovascular risk reduction sessions, observed and improved skills on minor surgery, gynecologic and articular infiltration techniques, always under supervision. It was also possible to contact briefly with the Residency Program of Family Medicine at Tufts University, participating in videoconferences and seminars, as well as on observation of patients and the discussion of clinical cases.

As conclusion, the author discusses the advantages of enrolling an international internship, such as the contact with new ideas and different ways of working and communicating, new skills (and their use as a tool for continuous improvement), the establishment of professional contacts and, last but not least, the valorization of the qualities of our own health system.

Keywords (MeSH): International Education Exchange; Primary Health Care. 